\title{
Análise de conceito sobre estilo de vida saudável no contexto da atenção primária de saúde
}

Concept analysis on healthy lifestyle in the context of primary health care

Análisis de concepto de estilo de vida saludable en el contexto de la atención primaria de salud

Joselane Izaquiel Marinho

ORCID: https://orcid.org/0000-0002-5713-016X Universidade Federal de Campina Grande, Brasil

E-mail: marinhojoselane96@gmail.com

Allana Petrúcia Medeiros de Miranda

ORCID: https://orcid.org/0000-0002-8228-3192 Universidade Federal de Campina Grande, Brasil E-mail:allanapetrucica@gmail.com

Anajás Silva Cardoso Cantalice ORCID: https://orcid.org/0000-0002-4709-2294 Universidade Federal de Campina Grande, Brasil E-mail:anajascardoso@gmail.com

Larissa Soares Mariz Vilar de Miranda ORCID: https://orcid.org/0000-0002-2021-1361 Universidade Federal de Campina Grande, Brasil E-mail:larissamariz@gmail.com

Matheus Figueiredo Nogueira ORCID: https://orcid.org/0000-0002-5787-7861 Universidade Federal de Campina Grande, Brasil

E-mail:matheusnogueira.ufcg@gmail.com

\begin{abstract}
Resumo
Objetivo: analisar o conceito de estilo de vida saudável no contexto da atenção primária de saúde. Metodologia: Tratase de uma análise de conceito proposta por Walker e Avant, modelo dividido em oito etapas, são eles: seleção do conceito; objetivos da análise conceitual; identificação dos possíveis usos do conceito; determinação dos atributos definidores; identificação do caso modelo; identificação de antecedentes e consequentes; e definição de referenciais empíricos. Resultado: Foi selecionado o conceito de estilo de vida saudável no contexto da APS, posteriormente, foi realizada uma revisão integrativa para identificar os possíveis usos do conceito, sendo eles: nas UBS, na comunidade, nas escolas, na família, no trabalho, nos parques, nas academias, no campo e nos ginásios; os atributos: prática de atividade física, alimentação saudável, não fumar, não ingerir bebidas alcóolicas, boa noite de sono, atuação da equipe multiprofissional, equilíbrio psicológico, fatores socioeconômico e determinantes de saúde; os antecedentes: construção de Políticas Públicas, ações assistenciais, educação em saúde, autonomia dos sujeitos, intervenções preventivas; e os consequentes: prevenção de doenças, qualidade de vida, bem-estar e longevidade. Conclusão: Diante da pesquisa realizada, foi possível o desenvolvimento de um conceito completo de estilo de vida saudável no contexto da APS. Percebe-se que na literatura o conceito foi abordado de forma fragmentada e simplificada. Portanto, este trabalho poderá ser norteado para o desenvolvimento de outras etapas do processo, fortalecendo o corpo científico da enfermagem, subsidiando o desenvolvimento de tecnologias para utilização do estilo de vida saudável no contexto da APS.
\end{abstract}

Palavras-chave: Análise de conceito; Estilo de vida; Pesquisa em enfermagem; Atenção primária de saúde.

\begin{abstract}
Objective: to analyze the concept of healthy lifestyle in the context of primary health care. Methodology: This is a concept analysis proposed by Walker and Avant, a model divided into eight steps, namely: concept selection; objectives of conceptual analysis; identification of possible uses of the concept; determination of defining attributes; identification of the model case; identification of antecedents and consequences; and definition of empirical references. Result: The concept of healthy lifestyle was selected in the context of PHC, later, an integrative review was carried out to identify the possible uses of the concept, namely: in UBS, in the community, in schools, in the family, at work, in the parks, gyms, countryside and gyms; the attributes: practice of physical activity, healthy eating, not smoking, not drinking alcohol, good night's sleep, performance of the multidisciplinary team, psychological balance, socioeconomic factors and health determinants; the antecedents: construction of Public Policies, assistance actions, health education, subjects' autonomy, preventive interventions; and the consequences: disease prevention,
\end{abstract}


quality of life, well-being and longevity. Conclusion: Based on the research carried out, it was possible to develop a complete concept of healthy lifestyle in the context of PHC. It is noticed that in the literature the concept was approached in a fragmented and simplified way. Therefore, this work can be guided for the development of other stages of the process, strengthening the scientific body of nursing, supporting the development of technologies for the use of a healthy lifestyle in the context of PHC.

Keywords: Concept analysis; Lifestyle; Nursing research; Primary health care.

\section{Resumen}

Objetivo: analizar el concepto de estilo de vida saludable en el contexto de la atención primaria de salud. Metodología: Se trata de un análisis de concepto propuesto por Walker y Avant, un modelo dividido en ocho pasos, a saber: selección de concepto; objetivos del análisis conceptual; identificación de posibles usos del concepto; determinación de atributos definitorios; identificación del caso modelo; identificación de antecedentes y consecuencias; y definición de referencias empíricas. Resultado: Se seleccionó el concepto de estilo de vida saludable en el contexto de la APS, posteriormente se realizó una revisión integradora para identificar los posibles usos del concepto, a saber: en UBS, en la comunidad, en las escuelas, en la familia, en el trabajo, en los parques, gimnasios, campo y gimnasios; los atributos: práctica de actividad física, alimentación saludable, no fumar, no beber alcohol, sueño reparador, desempeño del equipo multidisciplinario, equilibrio psicológico, factores socioeconómicos y determinantes de la salud; los antecedentes: construcción de Políticas Públicas, acciones asistenciales, educación en salud, autonomía de los sujetos, intervenciones preventivas; y las consecuencias: prevención de enfermedades, calidad de vida, bienestar y longevidad. Conclusión: a partir de la investigación realizada, fue posible desarrollar un concepto completo de estilo de vida saludable en el contexto de la APS. Se advierte que en la literatura se abordó el concepto de forma fragmentada y simplificada. Por tanto, este trabajo puede orientarse para el desarrollo de otras etapas del proceso, fortaleciendo el cuerpo científico de la enfermería, apoyando el desarrollo de tecnologías para el uso de un estilo de vida saludable en el contexto de la APS.

Palabras clave: Análisis de conceptos; Estilo de vida; Investigación en enfermería; Primeros auxilios.

\section{Introdução}

O Sistema Único de Saúde (SUS) no Brasil possui como principal porta de entrada a Atenção Primária à Saúde (APS) (Brasil, 2017), cujas ações e serviços são ofertados para toda a sociedade com vistas a assegurar o mais alto nível de saúde e bem-estar, envolvendo a promoção da saúde, prevenção de doenças, tratamento, reabilitação e cuidados paliativos de forma integral e que impacte positivamente na situação de saúde das coletividades (WHO, 2018). Embora a APS venha se desenvolvendo há décadas como sistema de saúde nacional no cuidado integral e coordenado com outros setores, ganhou visibilidade e destaque com a configuração e expansão da Estratégia de Saúde da Família, por seu modelo exitoso no acesso aos serviços e na implementação das diretrizes do SUS (Giovanella, 2019).

A visão da assistência em saúde em nível primário inclui a proteção da saúde da população como prioridade das sociedades e ambientes, atenção à saúde acessível e disponível, qualificada para tratar com respeito e dignidade e o envolvimento das pessoas na sua própria saúde. Assim a APS “é o enfoque mais eficaz, eficiente e equitativo para melhorar a saúde, o que faz dela um alicerce necessário para conseguir a cobertura universal de saúde” (OMS, 2019).

Dentre os cuidados prestados na APS, estão os relacionados à qualidade de vida, que consiste em uma percepção subjetiva do indivíduo em relação a sua posição na vida e a suas expectativas, padrões e preocupações (WHO, 1995). Trata-se de respostas individuais a saúde física, emocional e social e impacto da doença no seu cotidiano. Envolve o nível de independência, as relações sociais, as crenças pessoais e a relação com o meio ambiente. Dos comportamentos de risco que compõem a qualidade de vida está o estilo de vida (Malik, Weletti \& Hu, 2013).

O estilo de vida pode ser conceituado como um comportamento padrão que reflete as atitudes, os comportamentos e os valores que podem influenciar de forma positiva ou negativa o nível de saúde do indivíduo, e, dessa forma, sua qualidade de vida (Petroski \& Elegrine, 2009). No contexto atual, as mudanças demográficas, epidemiológicas, de trabalho e lazer, nas indústrias alimentícias e da globalização vem gerando mudanças significativas no estilo de vida e como consequência maior exposição a fatores de risco para desenvolvimento de doenças crônicas não-transmissíveis (DCNT) (Ferrari, Cesar, Alves, Barro, Goldbaum \& Fisberg, 2017). 
À vista disso, as doenças crônicas são um importante problema de saúde pública, ocasionando $68 \%$ das mortes no mundo. Destas, $40 \%$ são consideradas prematuras, ocorrendo antes dos 70 anos. As DCNT se atribuem $80 \%$ das consultas em Atenção Primária, 60\% das internações hospitalares e constituem a maior causa de incapacidade (Brasil, 2012). Os principais fatores de risco para o adoecimento por DCNT estão interligados ao estilo de vida da população como: tabagismo, consumo de álcool, alimentação não saudável e inatividade física, que podem ser modificados pela mudança de comportamento e por ações governamentais que regulamentem e reduzam, por exemplo, comercialização, consumo e exposição de produtos danosos à saúde (Brasil, 2020).

Em 2020 foi lançado pelo Ministério da Saúde o Plano de Ações Estratégicas para o Enfrentamento das Doenças Crônicas e Agravos não Transmissíveis no Brasil. Dentre as propostas está o fortalecimento das ações de saúde na APS e a melhoria da qualidade de vida, com vistas à mudança dos padrões alimentares, prática de atividade física, estilo de vida sedentário, hábito de fumar e consumir bebida alcoólica, entre outras (Brasil, 2020).

Para Almeida, Cassoti e Sena (2019), um estilo de vida é um sistema complexo formado por diversos elementos que se relacionam entre si e modifica-se e é modificado em um mesmo contexto de saúde. Reunir, portanto, as partes distintas do que é um estilo de vida em busca de um melhor estado de saúde, torna-se um desafio.

Nessa lógica, o conceito de estilo de vida saudável é construído de modo fragmentado na literatura e não permite uma visualização de sua natureza polissêmica e da amplitude que se deve alcançar a fim de prevenir e/ou minimização de danos em todas as faixas etárias no contexto da APS.

Conceitos são significados atribuídos a fenômenos e auxiliam na expansão e evolução do conhecimento da arte, além disso podem unificar a linguagem entre os profissionais de saúde fortalecendo o seu entendimento. Dessa forma, ressalta-se a importância de uma análise conceitual sobre o estilo de vida saudável no contexto da APS, favorecendo a classificação de conceitos úteis para a prática do cuidado em saúde e, de forma prática, a organização do conceito em questão poderá contribuir para subsidiar estratégias de promoção à saúde para a população, bem como os cuidados aos pacientes com DCNT e incentivo aos usuário da APS à responsabilização pela própria saúde, demonstrando a relevância do presente estudo.

Nessa perspectiva, o objetivo deste estudo consiste em analisar o conceito de estilo de vida saudável no contexto da Atenção Primária à Saúde.

\section{Metodologia}

\section{Tipo de estudo}

Consta uma Análise de Conceito, utilizada pelos investigadores com o objetivo de sintetizar e entender o conhecimento acerca de uma determinada área temática. (Zagonel, 1996; Fawcett, 2012). Conceitos são construções mentais, tentativas de requisitar estímulos ambientais em uma maneira significativa, são blocos que formam a base na construção de uma teoria. Analisar um conceito é útil nesse fenômeno, visto que facilitará a comunicação dos profissionais de enfermagem na atuação com usuários da atenção primária em saúde e a padronização da linguagem. Também a busca por promover o entendimento entre si sobre o fenômeno que está sendo discutido, facilitando as formas de reconhecimento e/ou medição desses conceitos em seus trabalhos (Walker \& Avant, 2010).

Para a análise de conceito, Walker e Avant (2010) recomendam oito etapas: seleção do conceito; objetivos da análise conceitual; identificação dos possíveis usos do conceito; determinação dos atributos definidores; identificação do caso modelo; identificação de antecedentes e consequentes; e definição de referenciais empíricos. Este é um processo mutável, devido à sua temporalidade. 
Assim, o conceito de interesse para esta análise é "estilo de vida saudável no contexto da atenção primária de saúde". A partir da seleção deste conceito, buscou-se refletir o tópico, sendo este importante e útil para o progresso teórico desta área, como aconselham Walker e Avant (2010).

\section{Coleta de dados}

O levantamento dos dados foi realizado nas seguintes bases de dados eletrônicas, nacionais e internacionais: Literatura Latino-Americana e do Caribe em Ciências da Saúde, (LILACS), Medical Literature Analysis and Retrieval System Online (MEDLINE), PubMed, na biblioteca eletrônica Scientific Electronic Library Online (SciELO) e pesquisa livre no Google Acadêmico. Como estratégia de investigação foram utilizados os descritores: "Life Style", "healthy" em inglês; "estilo de vida", "estilo de vida saudável" em português; "estilo de vida saludable" em espanhol; sendo combinados pelo operador booleano AND. As buscas realizadas ocorreram em dezembro de 2020.

Foram encontrados 37 artigos, sendo incluídos 11 artigos para esta revisão. Os critérios de inclusão considerados foram: estar disponível na íntegra, estudos primários, envolvendo seres humanos, sem custo para acesso, com resumo disponível, que abordassem o estilo de vida saudável em usuários da atenção primária de saúde, publicados nos idiomas português, inglês ou espanhol entre os anos 2015-2019. Após a leitura dos resumos excluíram-se 26 publicações por estarem repetidas nas bases de dados, por não incluir a APS, não abordar a temática em estudo, serem pagos e ou não estar dentro do intervalo de tempo delimitado.

Cada artigo foi lido na íntegra e revisto a fim de identificar os usos, atributos, antecedentes, consequentes e referências empíricas do conceito. Construiu-se uma tabela no Excel com todos os artigos e os dados coletados seguindo cada etapa do modelo de Walker e Avant (2010), explicados a seguir:

\section{Seleção do conceito}

O conceito deve refletir e ser orientado a partir de um problema de interesse e da vivência do pesquisador, requerendo cautela no momento da sua escolha. Isso porque quanto mais claros e concisos forem os conceitos, melhor o seu entendimento. Por isso, evita-se o uso de termos primitivos ou muito abrangentes os quais podem confundir a análise (Walker \& Avant, 2019). O conceito escolhido foi o estilo de vida saudável no contexto da APS e a questão de pesquisa que norteou a construção conceitual foi: Qual o conceito de estilo de vida saudável adotado nas publicações dos últimos 5 anos aplicado ao contexto da APS?

O conceito proposto nessa pesquisa surgiu diante da elaboração de um projeto de iniciação científica-CNPq sobre a temática, utilizando a Teoria Fundamentada no Dados, que requereu um entendimento aprofundado sobre os atributos e elementos antecedentes e consequentes do estilo de vida saudável no contexto da APS.

\section{Objetivos da análise conceitual}

Esta etapa refere-se à finalidade da análise conceitual, neste caso, trata-se da identificação do conceito de estilo de vida saudável no contexto da APS, possibilitando seu refinamento ou atualização para o contexto deste âmbito populacional (Walker \& Avant, 2010).

Esta análise com foco nos usuários da APS visa contribuir para a implementação de estratégias direcionadas a suprir as lacunas encontradas na literatura sobre o entendimento de estilo de vida saudável na APS. Esse conhecimento poderá favorecer a minimização de doenças emergentes e ascendentes na população através da implantação de programas, políticas públicas e parceria com a APS, considerando os atributos, características e particularidades que envolvem o estilo de vida 
saudável nestes usuários, trabalhando individualmente ou em conjunto os aspectos encontrados, de modo a prevenir ou minimizar as doenças relacionadas (DCNT) a inexistência de estilos de vida saudável. Além disso, permitirá à comunidade acadêmica conhecer o conceito de estilo de vida saudável neste contexto, instigando novas pesquisas que considerem os aspectos nele envolvidos.

\section{Identificação dos possíveis usos do conceito}

Nesta etapa realizou-se uma pesquisa bibliográfica, do tipo revisão da literatura (Botelho, Cunha \& Macedo, 2011). Além disso, realizou-se busca em dicionário e outras fontes científicas que abordassem o conceito de estilo de vida saudável (Walker \& Avante, 2010). A identificação dos possíveis usos do conceito permite encontrar como está sendo utilizado e aplicado, sendo recomendado buscar além da literatura da área da saúde, procurando em livros, dicionários e enciclopédias (Walker \& Avant, 2010).

\section{Determinação dos atributos definidores}

Verificou-se como cada atributo foi mencionado nos estudos e posteriormente a definição dos mais frequentes para melhor compreensão do conceito. Atributos definidores são palavras ou expressões que surgem repetidamente na literatura que mostram a essência do conceito, constituem características que expressam o conceito, evitando vieses na compreensão da sua natureza (Walker \& Avant, 2010; Fernandes, Nóbrega, Garcia \& Costa, 2011).

\section{Identificação do caso modelo}

Ocorre por meio da confecção de um caso baseado na realidade, que transmite características, atributos definidores que representam o conceito (Walker \& Avant, 2010). Demonstraram-se características definidoras, sendo apresentado um caso fictício construído com base nos resultados encontrados nos estudos da revisão integrativa e conhecimento prévio.

\section{Identificação de antecedentes e consequentes}

Antecedentes correspondem aos acontecimentos ou incidentes que ocorrem antes da existência do conceito, portanto não podem ser um atributo do conceito. Os consequentes são os acontecimentos que ocorrem como resultado do aparecimento do conceito (Walker \& Avant, 2010). Neste sentido, não se pode considerar que uma palavra seja, concomitantemente, o atributo, consequente e antecedente.

Após identificação dos atributos do conceito, procedeu-se nova leitura dos artigos na busca por eventos que ocorreram anterior ou como resultado da existência do conceito. Esses foram também transcritos para planilha Excel e compilada posteriormente.

\section{Definição de referenciais empíricos}

O pesquisador definiu nessa etapa como o conceito poderia ser mensurado. As referências empíricas são classes ou categorias de fenômenos reais que, quando presentes demonstram a ocorrência do conceito, possibilitando, assim, sua definição operacional. (Walker \& Avant, 2010).

Nesta etapa foram apresentados os instrumentos e métodos possíveis de determinação da existência do conceito estudado. Este passo da investigação foi guiado pela pergunta: Quais instrumentos de coleta de dados foram utilizados nos estudos da amostra que permitiram mensurar o conceito? Nesse estudo buscou-se localizar nos artigos escalas, questionários, padrões ou qualquer outro instrumento utilizado para avaliar o estilo de vida dos usuários da APS. 


\section{Resultados}

\section{Usos do conceito}

No material científico levantado foi possível identificar a demarcação de espaços para a o desenvolvimento de ações voltadas à promoção de estilos de vida saudáveis no contexto da APS, são eles: unidades básicas de saúde (Mendonça, Toled \& Lopes, 2015; Ferrari et al., 2017; Salami, Martelli, Robini \& Rochese, 2017; Madeira, Filgueira \& Nogueira, 2018; Almeida et al., 2018; Hafele \& Siqueira, 2019); nas escolas (Figueiredo, 2015; Ferrari et al., 2017; Almeida et al., 2018); na comunidade (Mendonça et al., 2015; Pôrto, Kumpel, Castro, Oliveira \& Alfiere, 2015; Figueiredo, 2015; Salami, 2017; Almeida et al., 2018; Saraiva, 2019; Hafele, \& Siqueira, 2019); na família (Figueiredo, 2015; Salami, 2017; Ferrari et al., 2017; Almeida et al., 2018; Toledo et al., 2017); no trabalho (Figueiredo, 2015; Toledo et al., 2017; Ferrari et al., 2017); nos parques (Figueiredo, 2015); nas creches (Almeida et al., 2018); nas academias (Toledo et al., 2017); no campo (Salami, 2017); e em ginásio (Saraiva, 2019).

\section{Atributos}

A partir da análise dos artigos pesquisados foram encontrados oito atributos sobre o estilo de vida saudável no contexto da APS, que caracterizam o estilo de vida saudável como: (1) prática de atividade física; (2) alimentação saudável; (3) atuação da equipe multiprofissional;(4) não fumar; (5) não ingerir bebidas alcoólicas; (6) qualidade do sono; (7) equilíbrio psicológico; e (8) determinantes de saúde.

$\mathrm{Na}$ análise dos artigos apresentada no Quadro 1 destacam-se a atividade física e os hábitos alimentares como dois elementos preponderantes do estilo de vida saudável. A alimentação adequada é indicada nos dados a partir de termos como: alimentação balanceada (Pôrto, 2015; Salami, 2017); padrão alimentar alterado (Figueredo, 2015); qualidade e quantidade dos alimentos ingeridos (Ferrari, 2017); alimentação como fenômeno da práxis dos profissionais de saúde da APS (Madeira, 2018); amamentação nos seis primeiros meses de vida (Piasetzki et al., 2018); e consumo de baixo teor de gordura (Almeida et al., 2018).

Esses dados incluem o estímulo, sensibilização e melhora dessas práticas desde a infância. Os pais ou responsáveis devem influenciar as crianças no sentido de promover bons hábitos alimentares e de atividade física, tendo em vista que a família é a figura central nesse contexto (Piasetzki et al., 2018).

A vista disso, no Brasil, a Política Nacional de Alimentação e Nutrição (PNAN) reposicionou a questão alimentar e nutricional na agenda das políticas públicas do setor saúde, enfatizando a importância de práticas alimentares e estilos de vida saudáveis como um componente importante para a promoção da saúde já nos primeiros anos de vida (Brasil, 2008), principalmente, na proteção e apoio à amamentação exclusiva até os seis primeiros meses de vida, e à alimentação complementar adequada em tempo oportuno (Piasetzki et al., 2018).

Grande parte dos artigos analisados sugerem que a participação dos profissionais da saúde é importante e representam impacto na mudança do estilo de vida. Essa atuação pode ser realizada a partir de atividades educativas (Mendonça et al., 2015; Piasetzki et al., 2018; Hafele \& Siqueira, 2019); orientações individuais e coletivas (Mendonça et al., 2015; Toledo, 2017; Hafele \& Siqueira, 2019); estratégias de intervenção (Mendonça et al., 2015; Ferrari, 2017); proteção e promoção da saúde (Toledo, 2017; Hafele \& Siqueira, 2019); e campanhas que sensibilizem mudanças comportamentais associadas a saúde (Saraiva, 2019).

Essas ações foram citadas preferencialmente para mudanças nos hábitos alimentares e de atividade física, mas podem ser aplicadas aos demais atributos do conceito. Para se ter uma dieta bem estruturada e organizada é necessário profissional. Da mesma forma, fazer exercícios com intuito de ajudar no novo estilo de vida parece ser mais efetivo quando orientado por uma 
equipe multiprofissional. Muitas das dietas feitas sem orientações não foram testadas e podem não funcionar, deixando o indivíduo sem motivação, o que o levaria a desistir de ter uma alimentação e estilo de vida saudável (Salami et al., 2017).

Observa-se, ainda, que os artigos destacam que uma grande parcela da população não segue um estilo de vida adequado em todas as faixas etárias (Pôrto et al., 2015; Salami et al., 2017; Häfele \& Siqueira, 2019). Isso porque um estilo de vida saudável não está limitado a prática de atividade física e uma alimentação balanceada. A complexidade do conceito está em associar esses elementos a fatores emocionais, sociais, culturais e de atuação da equipe de saúde.

O tabagismo e o consumo abusivo e/ou dependência de álcool são hábitos sociais adotados na maior parte da comunidade. O hábito de fumar e consumir álcool em excesso fazem parte da cultura da sociedade em que vivemos. Contudo, é importante destacar que são fatores modificáveis relacionados com o desenvolvimento de diversas doenças, como por exemplo, doenças cardiovasculares, obesidade, diabetes, entre outras (Mendonça et al., 2015; Ferrari et al., 2017).

Dentre os atributos do conceito é possível acrescentar a qualidade do sono (Salami et al, 2017; Almeida et al, 2018), onde recomenda-se dormir de 7 a 8 horas por dia, visto que durante o sono o corpo recupera-se de todo o esforço feito durante o dia (Salami et al., 2017). Os dados demonstram que ter repouso adequado, um sono adequado, faz parte de um estilo de vida saudável.

Outro atributo que recebe destaque nos artigos pesquisados foi o equilíbrio psicológico relacionado ao estresse (Pôrto, 2015). Parte da compreensão de que o desempenho emocional (Figueiredo, 2015) influencia no bem estar físico, mental, emocional, e também na prevenção de doenças, sendo estes muitas vezes relacionados ao tipo e quantidade de trabalho (Figueiredo, 2015; Salami et al., 2017).

Para o atributo de equilíbrio psicológico os artigos pesquisados mostram que é necessário ter visão otimista da vida, equilíbrio psicológico, saúde mental, apoio emocional, controle do estresse e desempenho emocional (Pôrto et al., 2015; Figueiredo, 2015; Almeida et al., 2018).

Apesar dos comportamentos, escolhas e decisões pessoais dos sujeitos integrarem os atributos do conceito de estilo de vida saudável no contexto da APS, estes sofrem influência direta de outro atributo citado nos artigos pesquisados: os determinantes de saúde.

George (2011) afirma que frequentemente os determinantes de saúde têm sido agrupados em 5 categorias: fixos ou biológicos (idade e sexo); os determinantes econômicos e sociais (estrato social e o emprego); os ambientais (qualidade do ar e da água, ambiente social); os de estilos de vida (alimentação, atividade física, tabagismo, álcool e comportamento sexual); e acesso aos serviços (educação, saúde, serviços sociais, transportes e lazer).

Assim, a partir da análise desse estudo percebe-se uma forte influência dos determinantes de saúde no conceito de estilo de vida saudável no contexto da APS, merecendo destaque os determinantes sociais e econômicos. Isso porque os artigos abordam no sentido de valores e oportunidades na vida, idade, sexo, etnia, gênero, condições de trabalho, oportunidades e fatores sociais, econômicos, histórico, político e cultural, moradia, renda, saneamento básico, educação, lazer, transporte, acesso a bens e serviços essenciais, relacionamentos e religiosidade.

Para Ferrati et al. (2017), as teorias atuais sobre estilo de vida saudável sugerem que as ações de saúde estão moldadas aos determinantes sociais de saúde. Madeira et al. (2018) acrescentam que os conceitos de EV estão intimamente ligados aos comportamentos individuais para promover saúde, sendo compreendida como fenômeno unicamente biológico. Contudo, fazse necessário acrescer a relação entre EV e saúde os determinantes sociais. 
Quadro 1: Atributos do 'estilo de vida saudável em usuários da APS' identificados nos artigos analisados, Cuité/PB, 2021.

\begin{tabular}{|c|c|}
\hline Artigos & Atributos \\
\hline $\begin{array}{l}\text { Pôrto, E. F. et } \\
\text { al., } 2015 .\end{array}$ & $\begin{array}{l}\text { Mudança nos hábitos de vida; Treinamento físico; Alimentação balanceada; Evitar o uso de álcool e fumo; Bom } \\
\text { relacionamento familiar e entre amigos; Prática de sexo seguro; Controle do estresse; Ambiente livre de estresse; Visão } \\
\text { otimista da vida; Valores e oportunidades na vida das pessoas; Manter controle do peso, do colesterol, da pressão } \\
\text { arterial, triglicérides e da glicemia; Ter repouso adequado; Equilíbrio psicológico; Alimentação equilibrada, uso de } \\
\text { carboidratos, proteínas, fibras, legumes e verduras; Evitar substâncias nocivas ao organismo; Recreação. }\end{array}$ \\
\hline $\begin{array}{l}\text { Figueiredo, J. P, } \\
2015 .\end{array}$ & $\begin{array}{l}\text { Determinantes sociais; Fatores extrínsecos; Alimentação equilibrada, diversificada e saudável; Necessidades } \\
\text { individuais; Atenção à idade, o sexo; Grau de atividade física; Contexto cultural (hábitos e costumes); Disponibilidade } \\
\text { de alimentos e locais para o consumo; condições de trabalho; Saúde mental; Apoio emocional; Desempenho emocional. }\end{array}$ \\
\hline $\begin{array}{l}\text { Mendonça, } \\
\text { R.D.; Toled, M. } \\
\text { T. T. \& Lopes, } \\
\text { A. C. S., 2015. }\end{array}$ & $\begin{array}{l}\text { Adoção de modos saudáveis de vida; Participação do profissional de saúde como educador; Orientações individuais ou } \\
\text { coletivas; Estratégias de intervenção. }\end{array}$ \\
\hline $\begin{array}{l}\text { Salami, A. D. } \\
\text { G., et al., } 2017 .\end{array}$ & $\begin{array}{l}\text { Estilo de vida menos sedentário; Aumento da procura por alimentos saudáveis; Oportunidades sociais; Boa alimentação; } \\
\text { Exercícios regulares; Evitar o consumo de substâncias nocivas ao organismo; Práticas saudáveis; Alimentação mais } \\
\text { balanceada; Adoção de um cardápio mais equilibrado; Atividade física; Padrão de sono. }\end{array}$ \\
\hline $\begin{array}{l}\text { Toledo, M. T. } \\
\text { T. et al., } 2017 .\end{array}$ & $\begin{array}{l}\text { Modos saudáveis de vida; Promoção de saúde; Redução da quantidade de alimentos processados e ultraprocessados; } \\
\text { Prática do aconselhamento. }\end{array}$ \\
\hline $\begin{array}{l}\text { Ferrari, T. K. et } \\
\text { al., } 2017\end{array}$ & $\begin{array}{l}\text { Atividade física; Escolaridade; Comportamentos individuais; Etnias; Gênero; Idade; Fatores sociais, culturais, } \\
\text { econômicos, históricos e políticos; Controle do tabagismo e do álcool. }\end{array}$ \\
\hline $\begin{array}{l}\text { Madeira, F. B. } \\
\text { et al., 2018. }\end{array}$ & $\begin{array}{l}\text { Construção de comportamentos e EV saudáveis; Contexto social; Fatores econômicos, políticos, ambientais e culturais; } \\
\text { Determinantes ou condicionantes de saúde; Alimentação como objeto das práxis de diversas profissões da área de saúde; } \\
\text { Alimentação saudável; Prática corporal e atividade física; prevenção e controle do tabagismo; Hábitos dietéticos e de } \\
\text { exercício; Moradia; Trabalho; Saneamento básico; Renda; Educação; Lazer; Transporte; Acesso aos bens e serviços } \\
\text { essenciais. }\end{array}$ \\
\hline $\begin{array}{l}\text { Piasetzki, C. T. } \\
\text { R; Bof, E. T. O., } \\
2018 .\end{array}$ & $\begin{array}{l}\text { Educação alimentar e nutricional; Formação de hábitos alimentares desde a infância; Amamentação nos primeiros seis } \\
\text { meses de vida. Determinantes sociais; Recursos financeiros; Tempo; Prática alimentar infantil; Preparação adequada de } \\
\text { alimentos; Atividade física. }\end{array}$ \\
\hline $\begin{array}{l}\text { Almeida, C. B. } \\
\text { et al., } 2018 .\end{array}$ & $\begin{array}{l}\text { Desenvolvimento positivo do estilo de vida e da saúde; Dietas com baixo teor de gorduras; Sono adequado; Prática de } \\
\text { exercícios aeróbicos; Hábito de não fumar; Práticas de atividades físicas regulares; Controle do estresse; Fator nutrição; } \\
\text { Atividades físicas; Comportamentos preventivos; Relacionamentos; Oportunidade na vida das pessoas; Religiosidade }\end{array}$ \\
\hline $\begin{array}{l}\text { Saraiva, P., } \\
2019 .\end{array}$ & $\begin{array}{l}\text { Promoção de EV saudáveis; Campanhas que possam provocar mudanças de comportamento associadas à saúde; } \\
\text { Divulgação da necessidade de hábitos mais saudáveis; Incorporação da atividade física no quotidiano de cada um nós; } \\
\text { Novos produtos alimentares; Prática desportiva. }\end{array}$ \\
\hline $\begin{array}{l}\text { Hafele, V. \& } \\
\text { Siqueira F. V., } \\
2019 .\end{array}$ & Ações de promoção e prevenção; Manutenção da saúde; Educação em saúde; Aconselhamento para a prática de AF. \\
\hline
\end{tabular}

Fonte: Dados da pesquisa (2021).

A partir da análise dos atributos do 'estilo de vida saudável no contexto da APS' é possível construir um conceito que será útil para unificação da linguagem dos profissionais de saúde e facilitará a comunicação para desenvolvimento de novas pesquisas à prática.

O Estilo de vida saudável no contexto da APS consiste primordialmente em cuidados relacionados a prática de atividade física e alimentação adequada em toda as fases da vida. Não obstante, trata-se de interromper os hábitos de fumar e ingerir bebidas alcoólicas, além de manter o padrão de sono regular e o equilíbrio psicológico. Todos estes elementos sofrem influência direta dos determinantes de saúde e devem ser regidos com auxílio da equipe multiprofissional que orienta, promove educação em saúde de forma individual e coletiva, elabora estratégias de intervenção e atua na promoção de hábitos próprios do EVS.

\section{Caso Modelo}

JPL, 66 anos, obeso, cardiopata e usuário da APS. Devido a evolução do seu quadro de obesidade, frequenta a Unidade Básica de Saúde do seu bairro regularmente. As complicações no seu estado de saúde derivam de uma vida sedentária e maus hábitos alimentares desde a infância, além do tabagismo e consumo frequente de bebida alcoólica ao longo da vida 
adulta. A família de JPL, o seu primeiro grupo social de contato, não tinha tempo de lhe preparar uma alimentação saudável e muito menos levá-lo ao parque para prática de atividade física. JPL não tinha horários fixos para a realização de suas atividades e os seus pais não se importavam em vê-lo por longas horas na frente do computador comendo alimentos ricos em gorduras. Por conta disso, JPL desenvolveu um quadro de obesidade o que o levou, na vida adulta, a fumar e a beber devido a um desequilíbrio psicológico gerado pela obesidade. Durante a adolescência, a equipe de saúde tentou intervir com medidas de prevenção contra agravamentos das DCNT, orientações em saúde para JPL e sua família, porém, eles não se importavam, levando ao agravamento do quadro com o passar do tempo. Quando adulto, mantinha um padrão irregular do sono, o que aumentou ainda mais os problemas de saúde. Assim, foi incentivado a procurar a UBS de seu bairro para receber auxílio da equipe multiprofissional. Foi preparado um plano terapêutico individual conforme cada profissional que JPL foi assistido (médico, enfermeiro, nutricionista e fisioterapeuta), visando a prevenção de agravos e promoção de estímulo à prática de atividade física, cessação do hábito de beber e fumar para que conseguisse dormir bem e se alimentar de forma saudável.

\section{Antecedentes}

Os antecedentes são fatos que ocorrem antes do estilo de vida saudável no contexto da APS. Nesta pesquisa, foram identificados cinco antecedentes: 1) construção de Políticas Públicas; 2) ações assistenciais; 3) educação em saúde; 4) autonomia dos sujeitos; e 5) intervenções preventivas.

A construção de políticas públicas e de ambientes que favoreçam escolhas saudáveis avança teoricamente, priorizando a promoção de saúde. Dá destaque ao fortalecimento da ação comunitária, à reorganização dos serviços de saúde com foco na atenção primária, e ao desenvolvimento de habilidades pessoais (Pôrto et al., 2015; Figueiredo, 2015; Piasetzki \& Bof, 2018).

Diversas políticas públicas e programas reconhecem e preveem a criação de ambientes favoráveis à saúde, que atendam às necessidades de saúde através de medidas intersetoriais, do empoderamento comunitário e do desenvolvimento de habilidades pessoais favoráveis à saúde em todas as etapas da vida (Figueiredo, 2015; Madeira et al., 2018). Por isso, torna-se primordial a realização de aconselhamento sobre modos saudáveis de vida pelos profissionais da saúde, especialmente sobre a prática regular de atividade física e alimentação saudável, tendo em vista seus comprovados benefícios (Mendonça et al., 2015).

Cabe destacar que na maioria dos artigos foi observada a preocupação de diferentes profissionais de saúde, em especial, para os comportamentos, hábitos de vida e costumes das pessoas como fatores principais no processo de saúde (Figueiredo, 2015; Mendonça et al., 2015; Ferrari et al., 2017; Almeida et al., 2017; Madeira et al., 2018; Saraiva, 2019). Logo, a participação do profissional de saúde como educador estimula reflexões e mudanças sobre comportamentos de interesse, envolvendo desde orientações individuais ou coletivas até estratégias de intervenção (Figueiredo, 2015; Ferrari et al., 2017; Almeida et al., 2017; Madeira et al., 2018).

Além disso, as escolhas de estilo de vida são comportamentos individuais que envolvem escolhas pessoais. Por essa razão, o estilo de vida relacionado à saúde também pode ser visto como consequência de uma escolha ou responsabilidade individual, e não como reflexo de uma contextualização social (Pôrto et al., 2015; Figuereido, 2015; Ferrari et al., 2017).

\section{Consequentes}

Os consequentes do estilo de vida saudável em usuários da APS incluem: prevenção de doenças, qualidade de vida, bem-estar e longevidade.

Após a adoção de estilos de vida saudáveis, como por exemplo, hábitos alimentares, atividade física regular, sono adequado, controle de peso, baixo consumo de álcool e de tabaco e o controle dos transtornos emocionais e psicológicos, espera-se que estas contribuam de alguma forma para a prevenção de futuros problemas de saúde (Figueiredo, 2015; Pôrto, et 
al., 2015; Toledo et al., 2017; Ferrari et al., 2017; Salami et al., 2017; Almeida et al., 2018; Madeira et al., 2018; Piasetzki \& Boff, 2018; Hafele \& Siqueira, 2019; Saraiva, 2019).

É de fundamental importância a promoção de estilos de vida saudáveis, como também a prevenção do desenvolvimento de comorbidades, garantindo a qualidade de vida das pessoas proporcionando a felicidade e o bem-estar (Mendonça et al., 2015; Figueiredo, 2015; Saraiva, 2019).

Estima-se que $75 \%$ dos novos casos de doenças crônicas poderiam ser preditas pelo tipo de dieta alimentar e o sedentarismo (Figuereido, 2015). Assim, o estilo de vida saudável pode levar a maior parte das pessoas a uma saúde positiva e com maior longevidade (Figueiredo, 2015; Almeida et al., 2018; Saraiva, 2019).

\section{Referencial Empírico}

Através da existência ou da presença dos referenciais empíricos, a ocorrência do conceito em si é demonstrada (Walker \& Avant, 2010).

Diante dos estudos analisados, não foi possível detectar instrumentos próprios para mensurar o estilo de vida saudável no contexto da APS. Contudo, segundo Walker \& Avant (2019) estes podem referir-se diretamente aos atributos definidores e, em muitos casos, podem ser idênticos.

Dessa forma, o referencial empírico desta pesquisa, por tratar-se de um fenômeno tanto objetivo quanto subjetivo, corrobora com os atributos por ser identificados através da menção do sujeito que decide adotar estilos de vida saudáveis ou por comportamentos compatíveis com a evidência do fenômeno, evitando a fragmentação desses conhecimentos e prezando-se pela compreensão e articulação entre eles.

\section{Discussão}

O conceito sugerido a partir desta pesquisa detecta atributos que inclui oito ações que, apesar de complexas, podem servir de base para as consultas dos profissionais de saúde voltadas ao contexto da APS: prática de atividade física, alimentação saudável, não fumar, não ingerir bebidas alcóolicas, boa noite de sono, atuação da equipe multiprofissional, equilíbrio psicológico e determinantes de saúde.

A indução da adoção de estilos de vida saudáveis reflete no reconhecimento da importância conferida a um modo de viver ativo como fator de proteção à saúde. Nesse contexto, profissionais da APS têm incluído informações e o aconselhamento das famílias sobre hábitos alimentares e prática de atividade física diária. Isso pode ser feito em diferentes contextos como escolas, comunidades ou unidades de saúde (Araújo et al., 2012). Contudo, o conceito de Estilo de vida saudável na APS ultrapassa as orientações limitadas a atividade física e alimentação e perpassa outros contextos de saúde como os aspectos psicológicos, culturais, econômicos, ambientais, sociais, religioso, político, afetivo, entre outros. O estilo de vida passa a ser um conceito amplo que depende das condições e oportunidades que as pessoas possuem (Almeida et al., 2018).

Compondo o conceito está a realização de atividade física e o lazer nos parques, nas academias e nos ginásios que também trazem qualidade de vida para a população, uma vez que, são determinantes para diferentes benefícios psicológicos, sociais e físicos bem como a redução do sedentarismo, do estresse, proporcionando saúde aos indivíduos (Szeremeta, 2009; Zannin, 2013).

Além disso também pode-se identificar como atributo do conceito os determinantes de saúde. O modelo de Dahlgreen \& Whitehead coaduna com os dados dessa pesquisa quando indica que o estilo de vida saudável é influenciado pelos determinantes sociais de saúde e contribuem para a exposição diferencial a fatores de risco à saúde como, por exemplo, o hábito tabágico e o sedentarismo. De igual modo o estilo de vida está interligado aos fatores pessoais (gênero, idade, fatores genéticos), interações sociais estabelecidas na comunidade (lazer, segurança, rede de apoio), condições de vida e de trabalho, 
disponibilidade de alimentos e acesso a serviços essenciais (desemprego, alimentação inadequada, habitações insalubres) e condições econômicas, culturais e ambientais. Esse modelo destaca-se os fatores não-clínicos como parte da situação de saúde da população e, dessa forma, como meio de se obter qualidade de vida e longevidade (Geib, 2012).

É importante perceber que a literatura aborda de forma limitada os fatores relacionados ao estio de vida saudável, e não revelam toda a sua complexidade e abrangência. Como exemplo podemos citar a pesquisa de Ferrari que ao avaliar o estilo de vida de das populações adolescente, adulta e idosa do Município de São Paulo, Brasil, partiu de cinco domínios: atividade física, consumo alimentar, tabagismo, consumo abusivo de álcool e dependência de álcool, e, exclui outros fatores citados acima no conceito proposto, como os socioeconômicos, culturais e políticos (Ferrari et al., 2017).

Todos os atributos do conceito apresentado só podem acontecer mediante fenômenos que antecedem o conceito como a implantação de políticas públicas que incentivem a construção, a revitalização de espaços percebendo a necessidade da população em aderir à estilos de vida mais saudáveis através do planejamento de ações educativas de acordo com a escolha de cada um dos sujeitos (Reis, 2001; Cohen et al., 2007; Cassou, 2009).

Salienta-se ainda, que cuidados de prevenção, tratamento das DCNT, intervenções de promoção à saúde, orientação sobre as mudanças da alimentação saudável bem como o incentivo à atividade física, desenvolvida pelos profissionais da saúde, tem repercutido diretamente na diminuição de morbimortalidade sendo de fundamental importância na APS (Fernandes, Conterato \& Mello, 2012).

Para Ferrari, as políticas públicas têm sido desenvolvidas no Brasil para incentivar ao estilo de vida saudável e estão implantadas no Brasil como o Pacto pela Saúde, o Pacto pela Vida, a Política Nacional de Promoção da Saúde, o Programa Academia da Saúde, a Política Nacional de Alimentação e Nutrição, o Programa Nacional de Controle ao Tabagismo e a Política de Atenção Integral em Álcool e Outras Drogas. No entanto, em sua pesquisa os resultados negativos relacionados fatores de alimentação, prática de atividade física, tabagismo, consumo de álcool e abuso de álcool, sugerem que essas políticas não estão sendo efetivas para melhorar o estilo de vida da população (Ferrari et al., 2017).

Como resultado do conceito construído estão os consequentes, que nessa pesquisa foi identificado com elementos como prevenção de doenças, qualidade de vida, bem-estar e longevidade. Para Sharkey (2001) manter um estilo de vida saudável tem impacto direto na qualidade de vida, que é compreendida como a percepção de bem-estar resultante de um conjunto de parâmetros individuais e socioambientais, modificáveis ou não, que caracterizam as condições em que vive o ser humano (Landeiro et al, 2011; Monteiro et al., 2010), o que reflete na redução de DCNT e na longevidade.

Desse modo, é possível perceber como atributos, antecedentes e consequentes se relacionam em um dado momento do processo de adoção de estilos de vida saudáveis. A ilustração teórica para este conceito pode ser observada na Figura 1: 
Figura 1 - Modelo conceitual para Estilo de vida saudável no contexto da APS.

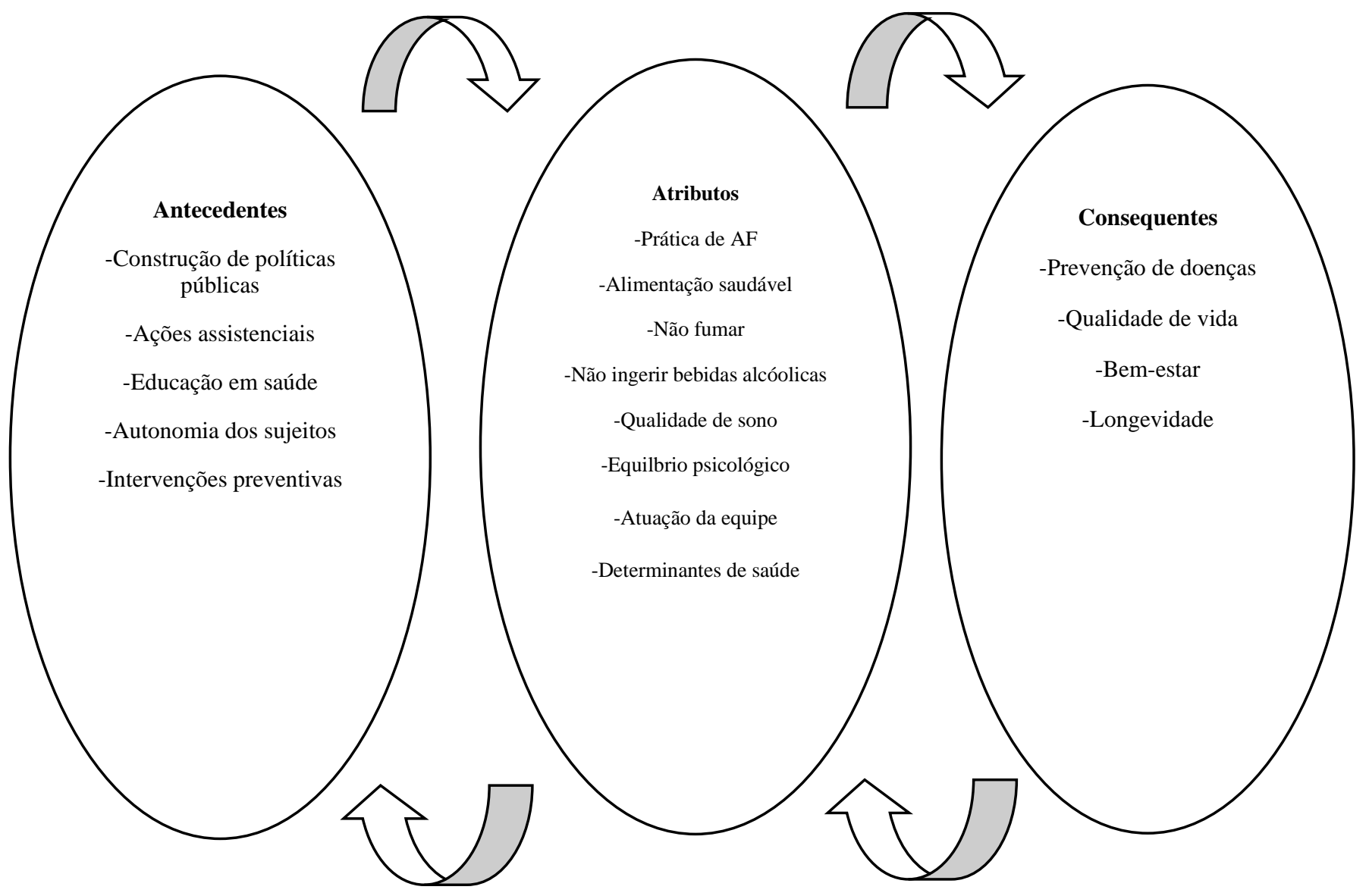

Fonte: Dados da Pesquisa (2021).

\section{Conclusão}

Diante da pesquisa realizada foi possível o desenvolvimento de um conceito completo de estilo de vida saudável no contexto da APS. Em sua completude, o conceito refere-se à prática de atividade física regular, alimentação saudável, preservação do hábito de não fumar e não ingerir bebidas alcóolicas, qualidade do sono, equilíbrio psicológico, atuação da equipe multiprofissional e determinantes de saúde. Isso antecedido por construção de políticas públicas, ações assistenciais, educação em saúde, autonomia dos sujeitos e intervenções preventivas, e, como resultado da aplicação do conceito a prevenção de doenças, qualidade de vida, bem-estar e longevidade. O conceito implementado de forma isolada e desassociada são ineficientes devido a sua complexidade, por isso, necessita de relações com os antecedentes e com os consequentes e em si mesmo. Assim, busca-se com isso uma complementaridade de elementos que se relacionam entre si, exercendo influência e sendo influenciado.

A análise do estilo de vida saudável no contexto da APS, auxilia na definição de um conceito a ser utilizado na prática, teoria, educação e pesquisa em saúde, e poderá subsidiar o desenvolvimento de ações para o aumento de práticas que promovam o estilo de vida saudável em situações de vulnerabilidade dos usuários e demostrem os impactos positivos que este hábito tem a curto e longo prazo.

Esta pesquisa, além de divulgar entre os profissionais de saúde a necessidade de priorizar estilos de vida saudáveis no contexto da APS, proporciona, através do conceito proposto, um auxílio aos profissionais na implementação de estilos de vida saudáveis na prática da atenção básica, bem como um destaque na importância da inclusão dos indivíduos nas escolhas por melhorias na qualidade de vida. 
Logo, sugere que novas pesquisas abordem essa temática, advindo novas evidências que possam ampliar as possibilidades de intervenções da saúde, melhorando a qualidade da assistência, bem como subsidiar na promoção de hábitos mais saudáveis.

\section{Referências}

Almeida, C. B., Casotti, C. A., \& da Silva Sena, E. L. (2018). Reflexões sobre a complexidade de um estilo de vida saudável. Avances en Enfermería, 36(2), $220-229$.

Araújo, S. N. M., Luz, M. H. B. A., da Rocha, S. S., da Silva, G. R. F., Duarte, M. R., \& de Sandes, N. M. (2012). Obesidade infantil: conhecimentos e práticas de enfermeiros da Atenção Básica. Enfermagem em foco, 3(3), 139-142.

Brasil. Ministério da Saúde. (2006). Portaria nº 648/GM, de 28 de março de 2006. Aprova a Política Nacional de Atenção Básica, estabelecendo a revisão de diretrizes e normas para a organização da Atenção Básica para o Programa Saúde da Família (PSF) e o Programa Agentes Comunitários de Saúde (PACS).

Brasil. Ministério da Saúde. (2012). Política Nacional de Atenção Básica. Programa Nacional de Melhoria do acesso e da qualidade da Atenção Básica (PMAQ).

Brasil. Ministério da Saúde. (2017). Portaria n 2.436, de 21 de setembro de 2017. Aprova a Política Nacional de Atenção Básica, estabelecendo a revisão de diretrizes para a organização da Atenção Básica, no âmbito do Sistema Único de Saúde (SUS). https://bvsms.saude.gov.br/b vs/saudelegis/gm/2017/prt2436_22_09_2017.html.

Brasil. Ministério da Saúde. (2020). Secretaria de Vigilância em Saúde. Departamento de Análise de Situação de Saúde. Plano de ações estratégicas para o enfrentamento das doenças crônicas não transmissíveis (DCNT) no Brasil2021-2030.

Botelho, L. L. R., Cunha, C. C. A., \& Macedo, M. O método de revisão integrativa nos estudos organizacionais. Gestão e Sociedade. 5(11): 121-36.

Cassou, A. C. N. (2009). Características ambientais, frequência de utilização e nível de atividade física dos usuários de parques e praças de Curitiba, PR.

Cohen, D. A., McKenzie, T. L., Sehgal, A., Williamson, S., Golinelli, D., \& Lurie, N. (2007). Contribution of public parks to physical activity. American journal of public health, 97(3), 509-514.

Fawcett, J. (2012). Thoughts on concept analysis: multiple approaches, one result. Nursing science quarterly, 25(3), $285-287$.

Ferrari, T. K., Cesar, C. L. G., Alves, M. C. G. P., Barros, M. B. D. A., Goldbaum, M., \& Fisberg, R. M. (2017). Estilo de vida saudável em São Paulo, Brasil. Cadernos de Saúde Pública, 33(1).

Fernandes, M. D. G. M., Nóbrega, M. M. L. D., Garcia, T. R., \& Macêdo-Costa, K. N. D. F. (2011). Análise conceitual: considerações metodológicas. Revista Brasileira de Enfermagem, 64, 1150-1156.

Giovanella, L., Mendonça, M. H. M. D., Buss, P. M., Fleury, S., Gadelha, C. A. G., Galvão, L. A. C., \& Santos, R. F. D. (2019). De Alma-Ata a Astana. Atenção primária à saúde e sistemas universais de saúde: compromisso indissociável e direito humano fundamental. Cadernos de saude publica, 35 , e00012219.

George, F. (2011). Sobre determinantes da saúde. Serviço nacional de saúde.

Geib, L. T. C. (2012). Determinantes sociais da saúde do idoso. Ciência \& Saúde Coletiva, 17, 123-133.

Häfele, V., \& Siqueira, F. V. (2019). Interventions with health professionals of primary care about physical activity counseling: sistematic review. Journal of Physical Education, 30.

Landeiro, G. M. B., Pedrozo, C. C. R., Gomes, M. J., \& Oliveira, E. R. D. A. (2011). Revisão sistemática dos estudos sobre qualidade de vida indexados na base de dados SciELO. Ciência \& Saúde Coletiva, 16, 4257-4266.

Malik, V. S., Willett, W. C., \& Hu, F. B. (2013). Global obesity: trends, risk factors and policy implications. Nature Reviews Endocrinology, 9(1), $13-27$.

Madeira, F. B., Filgueira, D. A., Bosi, M. L. M., \& Nogueira, J. A. D. (2018). Estilos de vida, habitus e promoção da saúde: algumas aproximações. Saúde e Sociedade, 27, 106-115.

Mendonça, R. D. D., Toled, M. T. T. D., \& Lopes, A. C. S. (2015). Incentivo à prática de aconselhamento sobre modos saudáveis de vida na Atenção Primária à Saúde. Escola Anna Nery, 19, 140-146.

Monteiro, R., Braile, D. M., Brandau, R., \& Jatene, F. B. (2010). Qualidade de vida em foco. Brazilian Journal of Cardiovascular Surgery, $25,568-574$.

Organização Mundial da Saúde (OMS) (2019). Declaração de Astana Genebra. OMS. https://www.paho.org/bra/in dex.php?option=com_ content\&view=article\&id=5711:declaracao-de-astana-sobre-atencao-primaria-a-saude-de-alma-ata-rumo-a-cobertura-universal-de-saude-e-o s-objetivos-dedesenvolvimento-sustentavel\&Itemid=0.

Petroski, E. L., \& Pelegrini, A. (2009). Associação entre o estilo de vida dos pais e a composição corporal dos filhos adolescentes. Revista paulista de pediatria, 27, 48-52.

Pôrto, E. F., Kümpel, C., de Castro, A. A. M., de Oliveira, I. M., \& Alfieri, F. M. (2015). Como o estilo de vida tem sido avaliado: revisão sistemática. Acta fisiátrica, 22(4), 199-205. 
Research, Society and Development, v. 10, n. 14, e321101422107, 2021

(CC BY 4.0) | ISSN 2525-3409 | DOI: http://dx.doi.org/10.33448/rsd-v10i14.22107

Reis, R. S. (2001). Determinantes ambientais para realização de atividades físicas nos parques urbanos de Curitiba: uma abordagem sócio-ecológica da recepção dos usuários (Doctoral dissertation, Universidade Federal de Santa Catarina, Centro de Desportos).

da Rosa Piasetzki, C. T., \& de Oliveira Boff, E. T. (2018). Educação alimentar e nutricional e a formação de hábitos alimentares na infância. Revista Contexto \& Educação, 33(106), 318-338. https://doi.org/10.21527/2179-1309.2018.106.318-338

Salami, A. D. G., Martello, J. C., Robini, E., Bocchese, P. A., \& Borelli, V. A. (2017). Estilo de Vida Saudável: Estudo do Comportamento dos Moradores de Antônio Prado. In: XVII Mostra de Iniciação Científica, Pós-graduação, Pesquisa e Extensão.

Saraiva, P. (2019). Promoção de estilos de vida saudáveis: uma distinção social? In Forum Sociológico. Série II (34), 81-88. CESNOVA.

Sharkey, B. J. (2001). Fitness and health. Champaign, IL: Human Kinetics (5a ed.).

Szeremeta, B., \& Zannin, P. H. T. (2009). Analysis and evaluation of soundscapes in public parks through interviews and measurement of noise. Science of the total environment, 407(24), 6143-6149.

Toledo, M. T. T. (2011). Adesão a modos saudáveis de vida por usuários de Serviço de Atenção Primária à Saúde mediante aconselhamento.

Walker, L. O., \& Avant, K. C. (2010). Strategies for theory construction in nursing (4a ed.). Pearson/Prentice Hall.

Walker, L. O., \& Avant, K. C. (2019). Strategies for theory construction in nursing (6a ed.). Pearson/Prentice Hall.

Whittemore, R., \& Knafl, K. (2005). The integrative review: updated methodology. Journal of advanced nursing, 52(5), 546-553.

Whoqol Group. (1995). The World Health Organization quality of life assessment (WHOQOL): position paper from the World Health Organization. Social science \& medicine, 41(10), 1403-1409.

World Health Organization and the United Nations Children's Fund (UNICEF), de 2018, https://apps.who.int/iris/bitstream/handle/10665/328065/WHO-HISSDS-2018.15-eng.pdf?sequence=1\&isAllowed=y.

Zagonel, I. P. S. (1996). Análise de conceito: um exercício intelectual em enfermagem. Cogitare Enfermagem, 1(1).

Zannin, P. H. T., Engel, M. S., Fiedler, P. E. K., \& Bunn, F. (2013). Characterization of environmental noise based on noise measurements, noise mapping and interviews: A case study at a university campus in Brazil. Cities, 31, 317-327. 